BULLETIN Bulletin hispanique

HISPANIQUE Université Michel de Montaigne Bordeaux

$118-2 \mid 2016$

Varia

\title{
La versión del Sueño de la muerte en Desvelos soñolientos y verdades soñadas
}

Irene Bertuzzi

\section{(2) OpenEdition}

1 Journals

Edición electrónica

URL: http://journals.openedition.org/bulletinhispanique/4507

DOI: 10.4000/bulletinhispanique.4507

ISSN: 1775-3821

Editor

Presses universitaires de Bordeaux

Edición impresa

Fecha de publicación: 15 diciembre 2016

Paginación: 473-492

ISBN: 979-10-300-0125-9

ISSN: 0007-4640

Referencia electrónica

Irene Bertuzzi, «La versión del Sueño de la muerte en Desvelos soñolientos y verdades soñadas », Bulletin hispanique [En línea], 118-2 | 2016, Publicado el 15 diciembre 2019, consultado el 30 diciembre 2019.

URL : http://journals.openedition.org/bulletinhispanique/4507; DOI : 10.4000/bulletinhispanique.4507 


\title{
La versión del Sueño de la muerte en Desvelos soñolientos y verdades soñadas
}

\author{
IRENE BERTUZZI \\ Universidad de Santiago de Compostela
}

Le texte du Sueño de la muerte contenu dans l'édition Desvelos soñolientos y verdades soñadas (Zaragoza, 1627) présente de nombreux et profonds changements par rapport à la version de la première édition Sueños y discursos (Barcelone, 1627). Après avoir analysé les variantes du texte de Desvelos l'auteur émet l'hypothèse que c'est à Lorenzo Van der Hammen plutôt qu'à Quevedo qu'il convient d'imputer la nouvelle version du Sueño de la muerte.

Mots-clés: Desvelos soñolientos, Sueños y discursos, Quevedo, Lorenzo van der Hammen, critique textuelle.

El texto del Sueño de la muerte contenido en la edición Desvelos soñolientos y verdades soñadas (Zaragoza, 1627) presenta numerosos y profundos cambios con respecto a la versión de la primera edición Sueños y discursos (Barcelona, 1627). A través del análisis de las lecturas privativas del texto de Desvelos este artículo propone la hipótesis de que la revisión del Sueño de la muerte parece imputable a Lorenzo Van der Hammen antes que a Quevedo.

Palabras clave: Desvelos soñolientos, Sueños y discursos, Quevedo, Lorenzo van der Hammen, crítica textual.

The text of the "Sueño de la muerte» in the edition "Desvelos soñolientos y verdades soñadas» (Zaragoza, 1627) presents deep and numerous modifications in relation to the version contained in the first edition «Sueños y discursos» (Barcelona, 1627). Through the analysis of the variants of the text of "Desvelos", this paper proposes the hypothesis that Lorenzo Van der Hammen, and not Quevedo, was responsible for this new version of the «Sueño de la muerte».

Keywords: Desvelos soñolientos, Sueños y discursos, Quevedo, Lorenzo van der Hammen, textual criticism 
$\mathrm{E}^{\mathrm{n}}$ n el ámbito de los estudios dedicados a la compleja transmisión textual de la obra de Francisco de Quevedo conocida como los Sueños, se ha prestado poca atención a la edición que lleva el título Desvelos soñolientos y verdades soñadas $(D)$, por lo que a día de hoy se desconoce el nivel de implicación del autor en su preparación ${ }^{1}$. Desvelos fue publicada por Pedro Vergés, a costa de Roberto Duport, en Zaragoza en $1627^{2}$, el mismo año en el que salió la primera edición de la obra, Sueños y discursos de verdades descubridoras de abusos, vicios y engaños, en todos los oficios y estados del mundo, impresa en Barcelona $\left(B_{27}\right)^{3}$. Con respecto al impreso barcelonés, $D$ conserva algunas variantes de los testimonios manuscritos que no están en la princeps, pero también presenta numerosas e importantes lecturas privativas que se concentran, sobre todo, en el Sueño de la muerte. La entidad de tales variantes -entre las cuales se registran adiciones, cambios en la estructura del periodo, alteraciones en el orden de los párrafosmuestra que el texto fue sometido a una profunda y cuidadosa revisión, por lo que cabe la posibilidad de que Desvelos contenga una nueva versión de la obra atribuible, parcial o completamente, al autor.

En el breve texto preliminar El librero al lector, Duport declara que Quevedo no supervisó la edición de la obra: «los yerros que encontrares los atribuyas no hallarse presente, con que el cuidado y vigilancia que he puesto en procuralla me anima a pedirte los perdones». Sin embargo, la carta nuncupatoria de Lorenzo Van der Hammen ${ }^{4}$, vicario de Júbiles, dirigida al capellán de su majestad Francisco Jiménez de Urrea, insinúa que los manuscritos en los que se basó la edición proceden del mismo Quevedo:

Remito a vuestra merced esos Sueños del amigo, como prometí, y le aseguro se pueden ahora leer sin escrúpulo, porque los he corregido por los originales que en mi librería tengo, y aun yo mismo he escrito gran parte, como lo dirá la letra. Por ellos verá cómo es cierto lo que le afirmé, y cuán faltos están esotros, llenos de yerros y con

1. Beatriz González López se ocupó de Desvelos hace años, en su tesina de licenciatura (González López 1994) dedicada a la edición barcelonesa de los Sueños de 1628, en la que se centró en el Sueño de la Muerte, y en un artículo titulado «Hacia una edición de los Sueños: Desvelos soñolientos» (González López 1999), que sentaron las bases para una futura profundización en el análisis de este testimonio; asimismo tuvieron el mérito de destacar la presencia de posibles variantes de autor en Desvelos. Sin embargo, algunas de sus consideraciones resultan inexactas a causa de que en su estudio no tomó en consideración los testimonios manuscritos. De hecho, si bien es verdad que el impreso presenta importantes lecturas privativas, algunas de las variantes que se citan como exclusivas de $D$ proceden, en realidad, de los manuscritos.

2. En la portada se menciona que la edición posee licencia, aunque esta no se incluye entre los preliminares legales. En efecto, la edición cuenta solamente con la aprobación, que lleva la fecha 31 de mayo de 1627. También en la portada se lee que el texto ha sido "Corregido y enmendado agora de nuevo por el mismo autor» y que el volumen incluye "un tratado de la Casa de locos de amon, obra que se suele considerar apócrifa (Jauralde Pou 519-520).

3. En 1627 se publicaron otras dos ediciones de la obra, una en Valencia y la otra en Zaragoza, que llevan el mismo título de la edición príncipe y derivan directamente de aquella.

4. «Lorenzo van der Hammen y León (1589-1664), hermano mayor de Juan, estudió en la Universidad de Alcalá de Henares, y prosiguió su carrera eclesiástica en Granada y Madrid. Participó igualmente en la vida intelectual de la Corte. Amigo de Quevedo, Lope de Vega y Juan Pérez de Montalbán, fue muy estimado por su saber y sus virtudes» (Véliz y García Valverde 3). 
mil convicios. La culpa ha tenido este caballero (como siempre le he advertido) en dejarlos trasladar, pues cada uno ha quitado y puesto según su antojo lo que más bien le ha parecido; y en particular los que ganan de comer en esta corte con este género de trabajo [...] Los que se precian de vanos y se pierden por andar impresos sus nombres, ya hubieran dado a la estampa estas vigilias, pero su modestia no lo ha permitido, aunque con daño de su reputación. (Van der Hammen 2007: 556)

Cuando en 1625 Van der Hammen publicó por vez primera su obra Don Felipe el prudente, segundo de este nombre, rey de las Españas y Nuevo Mundo, el libro incluía una carta de aprobación de Quevedo 5 . Parece que a raíz de esto los dos iniciaron una relación continuada (Jauralde Pou 498), atestiguada, entre otras cosas, por el hecho de que Van der Hammen fuese quien se ocupó de escribir el prólogo de la primera edición de Política de Dios autorizada por su autor (Madrid 1626) 6 .

En cuanto a Desvelos, según lo que se lee en la carta nuncupatoria, Van der Hammen debió de recibir un texto de los Sueños que luego corrigió, basándose en los «originales» que supuestamente poseía, y, además, confiesa haber insertado él mismo unos cambios. El amigo de Quevedo afirma también que el autor dejó circular su texto sin control y que no se preocupó nunca de publicarlo: «su modestia no lo ha permitido, aunque con daño de su reputación». Sin embargo, la carta no está fechada y no sabemos si es anterior o posterior a la impresión de $B_{27}$. La fecha de redacción de la carta sería determinante para saber si Van der Hammen se está refiriendo a que las anteriores ediciones de los Sueños se publicaron sin la complicidad de su autor.

Estas afirmaciones constituyen un punto de partida para reflexiones diversas sobre la naturaleza del texto contenido en la edición de $D$ y sobre la implicación de Quevedo en ella. Empezando por este aspecto, siendo Van der Hammen amigo y, en ocasiones, colaborador de Quevedo, es probable que poseyera unos textos de los Sueños que el autor mismo le habría dejado, los que él llama «originales». Sin embargo, de acuerdo con sus palabras existe otro texto que cayó en sus manos y que él se preocupó de corregir. Es posible que este texto y la tarea de corregirlo se la hubiera encomendado Ruberto Duport, después de haberse procurado una copia de los Sueños. En principio, parece poco probable que el mismo Quevedo hubiese proporcionado a Duport una copia de la obra, porque, de ser así, no se explicaría la necesidad de someterla a un control con los «originales». Quizás lo que hizo Quevedo fue dar a Van der Hammen su permiso para colaborar con Roberto Duport en la publicación de los Sueños.

5. En su texto preliminar Quevedo (1633: 4) exalta sobre todo la brevedad y autenticidad con las que Van der Hammen ha sabido resumir la vida y los hechos de Felipe II: "Con tal estudio y diligencia ha desembarazado vuestra merced de la prolixidad de los volúmenes de la historia de Filipe Segundo, que le estamos agradecidos con toda estimación a que haya abreviado la vida de aquel monarca [...] Vuestra merced, docto, fidedigno y modesto, deja vivir su vida al príncipe, y quiere que se lea lo que fue, no lo que quiere que crean o lo que quisiera que hubiera sido».

6. Van der Hammen compuso también la aprobación para la edición de Quevedo de la poesía de fray Luis de León (fechada en 1629): «Esta aprobación constituye la última evidencia de la relación amistosa entre Quevedo y Lorenzo van der Hammen» (Véliz y García Valverde 11). 
Es una hipótesis verosímil si se considera la serie de obras quevedianas editadas por Duport. Previamente a la edición de Desvelos, Roberto Duport se había ocupado de hacer imprimir Política de Dios y el Buscón (ambos en Zaragoza en 1626, en la imprenta de Pedro Vergés). Si en el caso de Política de Dios el mismo Duport afirmó que la obra había sido publicada sin el consentimiento de Quevedo (Díaz Martínez 167), la implicación del autor en el Buscón ha dividido a los críticos: Fernando Lázaro Carreter (XVI-XX) negó la participación de Quevedo en la edición príncipe de esta obra, idea que los estudiosos asumieron y aceptaron, hasta que Alfonso Rey opinó que hay razones para creer lo contrario ${ }^{7}$. De todas maneras, si es verdad que Duport publicó Política de Dios sin el consentimiento del autor, esto no excluye que en ocasiones posteriores los dos hayan colaborado de manera más o menos directa: «es difícil negar la existencia de algún tipo de entendimiento entre Quevedo y Duport, a juzgar por la lista de obras que van a seguir imprimiéndose en los años sucesivos» (Rey 169). Además de Política, el Buscón y Desvelos, en 1629 Duport edita El peor escondrijo de la muerte, Memorial por el patronato de Santiago y Cuento de cuentos; en 1630, Doctrina moral y El chitón de las tarabillas (Rey 169).

En lo relativo a las características de la obra editada en Desvelos, a nivel general, las primeras diferencias que se observan con respecto a las demás ediciones de los Sueños están, evidentemente, en el título, y en el hecho de que el volumen contiene solo tres de las cinco partes que forman el conjunto. El título aparece notablemente abreviado: en vez de sueños se habla de «desvelos sońolientos», mientras que se mantiene la palabra verdades, pero acompañada solamente de soñadas. En lo que atañe al contenido, Desvelos no incluye los «discursos», es decir El alguacil endemoniado y El mundo por de dentro, sino solo los tres «sueños», que aparecen con el orden alterado: Sueño de la muerte, El sueño del Juicio final y Sueño del infierno ${ }^{9}$. Además, la dedicatoria a doña Mirena Riqueza, texto que en los demás testimonios precede el Sueño de la muerte, en $D$ encabeza el conjunto de la obra y constituye el único preliminar literario.

La alteración del orden de los «sueńos», que en $B_{27}$ reproduce la cronología de la redacción de las piezas, podría deberse a motivos diversos. Según afirma

7. En 1626 «Quevedo acompaña al rey en el demorado viaje a sus reinos de la Corona de Aragón. Zaragoza y Barcelona son ciudades visitadas en la jornada real. Se ha discutido sobre la posible intervención de Quevedo en la publicación zaragozana de dos de sus obras, a pesar de que su editor, Roberto Duport, reclama la iniciativa. Sin duda, es probable que el viaje de Quevedo aumentase el interés por sus obras y favoreciese la búsqueda y difusión de copias manuscritas. Incluso las obras escritas durante el mismo viaje circularon inmediatamente» (Moll 9).

8. González López (1999: 159) opina que, aunque sea imposible establecer el origen del epígrafe, «su tono parece "fuertemente quevedesco"», mientras que Ilse Nolting-Hauff (57) lo encuentra "vago y seguramente no auténtico». Creo que el título de por sí no ofrece indicios claros sobre su autoría.

9. González López (1999: 160) hace notar que «Alguacil y Mundo, ausentes en D, presentan una ambientación distinta; su modelo de narración es el del coloquio entre narrador y personaje. La distinta denominación perseguiría reflejar una diferente unidad estructural, determinada, a su vez, por la unidad temática de las obras que incluye». 
Crosby en el apartado dedicado a Desvelos en la descripción de las ediciones de los Sueños incluida en su edición de la obra, pudo haber influido en la decisión el hecho de que Muerte es la parte que se modificó más con respecto a las otras dos: «Es posible que el motivo de la alteración en el orden clásico responda simplemente al deseo de colocar en primer lugar el sueńo cuyo texto ostenta mayores "mejoras" en comparación con las otras ediciones a la venta en aquel entonces» (Quevedo 1993: 783). Otra posibilidad es que se haya preferido seguir el orden de las postrimerías: muerte, juicio, infierno, y que el hecho de que las modificaciones se centrasen en el primer «sueño» sea una consecuencia del desplazamiento en vez de la causa.

El presente artículo aborda solo el análisis de uno de los tres "sueños» de la edición de Desvelos: el Sueño de la muerte, que ha sido elegido por ser el que sufrió la mayor cantidad de modificaciones. El primer paso del estudio textual de esta parte de la obra consiste en intentar descubir la procedencia del texto que sirvió para preparar la edición de Desvelos, o, por lo menos, averiguar de qué manera $D$ se relaciona con los testimonios de los que disponemos. Esto permitirá aislar las lecturas privativas de $D$ para poder analizarlas aparte. Posteriormente, dado que Van der Hammen afirma haber retocado el texto («aun yo mismo he escrito gran parte»), en primer lugar será necesario establecer en qué medida las variantes introducidas en $D$ pueden atribuirse a él. En segundo lugar, habrá que considerar la posibilidad de la presencia de variantes de autor, porque, dada la relación de amistad entre Quevedo y Van der Hammen, no es inverosímil que el autor le dejara una copia de la obra («los originales que en mi librería tengo») que contuviera cambios insertados por él mismo.

\section{La RELACión de $D$ CON $B_{27}$ Y CON LOS TESTIMONIOS MANUSCRITOS}

Al comparar la versión de Muerte de $D$ con la de cada uno de los testimonios manuscritos que se conservan (llamados $A_{b}, H_{l}, J, P$ y $Q$ ) ${ }^{10}$ y con la edición príncipe, es evidente que su texto se acerca mucho más al contenido de los primeros que al del impreso. Las variantes compartidas de manera exclusiva con $B_{27}$ son, de hecho, muy escasas, sobre todo considerando la amplitud de la pieza.

Sin duda, la coincidencia más importante entre las dos ediciones es la que se encuentra en el texto de la dedicatoria a Mirena Riqueza, que en todos los manuscritos (excepto $J$ que carece de él) y en $B_{27}$ precede al Sueño de la muerte, mientras que en $D$ se encuentra al principio del libro, a modo de texto preliminar de toda la obra:

10. Las siglas $J, P$ y $Q$ son las mismas que Crosby asignó a estos tres manuscritos en su edición de los Sueños ( $c f$. Quevedo 1993: xvii-xix); mientras que los testimonios llamados $A_{1}$ y $H_{1}$ son de descubrimiento reciente, por lo que el estudioso americano desconocía su existencia (sobre estos dos manuscritos ver Milagros Rodríguez Cáceres 2007). 
Procurado he pedir el estilo y sazonar la pluma con curiosidad D $\mathrm{B}_{27}$

Procurado he pulir el estilo y sazonar la pluma con curiosidad $\mathrm{A}_{1} \mathrm{H}_{1} \mathrm{PQ}$ Procurado] procurando $\mathrm{H}_{1}$ pulir] polir $\mathrm{A}_{1} \mathrm{H}_{1}$ pluma] cura $\mathrm{P}$ (Sources) Ce sont les manucrits mêmes, je dis ça dans le texte et la note 10

Desvelos reproduce el mismo error de copia que se cometió en $B_{27}$ : la sustitución de pulir por pedir, verbo que carece de sentido en la frase. Me parece que el error tiene valor conjuntivo y demuestra que la dedicatoria de $D$ fue tomada del impreso barcelonés o de un antecedente manuscrito de $B_{27}$. En el texto de Muerte no se encuentran otros errores en los que $D$ coincida con $B_{27}$; además, las variantes compartidas por estos dos testimonios son mínimas e insignificantes. Si se excluyen las lecturas privativas de $D$, su texto coincide casi siempre con el de la mayoría de los testimonios manuscritos: frente a los errores de $B_{27}, D$ lee como los manuscritos; donde el texto está censurado en $B_{27}, D$ mantiene la lectura original; finalmente, tampoco aparecen en $D$ las adiciones de $B_{27}$ (ni las breves, ni las más extensas).

Por ejemplo, el texto de Desvelos coincide con los manuscritos en algunas variantes que parecen mejores lecturas que las que se encuentran en B27 (compartidas, en los casos citados, también por $J$ ):

[médicos:]

Eran estos gran número, y todos venían chorreando platicantes $\mathrm{DA}_{1} \mathrm{H}_{1} \mathrm{Q}$ chorreando] choriando Q

Eran estos en gran número, y todos rodeados de platicantes $\mathrm{B}_{27} \mathrm{~J}$ en] om. J

Eran estos en gran número, y todos venían manando platicantes $\mathrm{P}$

[barberos:]

entretúveme en verlos manosear una cara y sobajar una zalea, y lo que se huelgan con un testuz en el lavatorio $\mathrm{DA}_{1} \mathrm{H}_{1} \mathrm{PQ}$ zalea] zulea $\mathrm{H}_{1}$ una cabeza $\mathrm{P}$ testuz] restituem (?) $\mathrm{H}_{1}$ testuzo $\mathrm{Q}$ el] un $\mathrm{Q}$

entretúveme en verlos manosear una cara, sobajar otra, y lo que se huelgan con un testuz en el lavatorio $B_{27}$ s sobajar] y sobajar J

En ambos casos la variante de $B_{27}$ y $J$ es aceptable, porque encaja perfectamente en el sentido de la frase. Sin embargo, en el primer ejemplo hay razones para preferir la lectura de la mayoría de los testimonios manuscritos y de $D$ frente a la de $B 27$ y $J$, dado que el verbo chorrear asociado a objetos «no líquidos» es una imagen a la que Quevedo recurre en otros lugares, como señala Lía Schwartz (1983: 172). En El chitón de las tarabillas se lee: «cual andas por los corrillos chorreando libelos y en las conversaciones rebosando sátiras, empreñando las esquinas de cedulones» (Quevedo 2005: 201); asimismo, en el Discurso de todos los diablos, en el que se dice del entremetido: «venía vaciándose de palabras y chorreando embustes» (Quevedo 2003a: 492); y, finalmente, en La Hora de todos, donde la mujer afeitada se describe como: «hecha un infierno, chorreando pantasmas» (Quevedo 2003b: 619). A la luz de los lugares paralelos señalados, lo más probable es que la variante presente en el texto original fuese 
la que se encuentra en la mayoría de los manuscritos y en $D$. Además, la lectura «venían chorreando» se adapta al usus scribendi $i^{11}$ quevediano, donde «El uso de un adyacente ilógico o sorprendente en relación con el vocablo al que califica es un conocido cauce de agudeza, y proporciona al conjunto de la expresión un agudo valor metafórico» (Azaustre Galiana 166).

En el segundo ejemplo, las tres variantes ofrecidas por los testimonios son aceptables. Es claro el significado de la versión que ofrecen $B_{27}$ y $J$ (que leen otra), y $P$ (cabeza). En cuanto a zalea - «la piel del carnero seca con lana, y sin curtir» (Autoridades)-, puede intepretarse como metáfora de barba. Quevedo la emplea de esta manera en La hora de todos ${ }^{12}$, texto en el que se encuentra la expresión «la zalea jurisconsulta» (Quevedo 2003b : 607). La palabra se utiliza con el mismo valor metafórico en el romance Cansado estoy de la corte: «Un abogado, que quiere, / por barbado, corregir, / con más zalea que leyes, / menos textos que nariz» (Quevedo 1969-1981: n 749, vv. 29-32).

En cuanto a la relación entre Desvelos y los manuscritos, es imposible saber si $D$ tiene algún tipo de vinculación con alguno de los que hoy se conservan, porque no se ha encontrado ningún error conjuntivo, ni tampoco ninguna variante, que permita al menos acercar la versión de la edición zaragozana a una u otra rama de la transmisión manuscrita de Muerte. Cabe destacar una variante que el impreso comparte con el manuscrito $H_{1}$ frente a $B_{27}$ : se trata de un añadido que amplía el grupo de personajes procedentes de refranes ${ }^{13}$. Este ańadido está ubicado en la parte de texto que se conserva solamente en los testimonios manuscritos $A_{l}$ y $H_{1}$ y en el impreso $B_{27}$ (los manuscritos $J$, $P$ y $Q$ están incompletos); de ellos, $H_{1}$ es el único que contiene la adición. No obstante, el hecho de que $D$ coincida con $H_{1}$ en esta variante no resulta significativo a la hora de establecer una relación entre estos dos testimonios, porque no comparten ninguna otra variante significativa de manera exclusiva.

Esta adición parece atribuible a Quevedo; de hecho, antes del descubrimiento de $H_{1}$, cuando se creía que era lectura privativa de $D$, su presencia llevó a sospechar que el autor había intervenido en la edición zaragozana ( $c f$. González López 1999: 162). Se reproduce a continuación el primer párrafo de la adición:

Dando gritos y alaridos venían a un muerto diciendo: «¡A mí me toca!, ¡yo lo sabré!, ¡ello dirá!, entenderémonos, ¿qué es esto?» y otras razones. "¿Quién es este tan entremetido en todas las cosas?» dije; y respondió un difunto: «Este es Vargas, que como dicen "averígüelo Vargas", viene averiguándolo todo». Topó en el camino a Villadiego; el pobre estaba afligidísimo, hablando entre sí. Llamole y dijo: «Señor Vargas, pues vuestra merced lo averigua todo, hágame merced de averiguar quién fueron las de Villadiego, que todos las toman, porque yo soy Villadiego, y en tantos ańos no lo he

11. Con el término usus scribendi me refiero al estilo del autor y de su época, desde la "perspectiva [...] de la retórica clásica, es decir, de la inventio, la dispositio y la elocutio de un autor y de su tiempo» (Blecua 124).

12. Lo señala Crosby en la nota explicativa que acompaña a este pasaje en su edición de los Sueños (Quevedo 1993 : 1402).

13. En $H_{1}$ la adición empieza en el folio 90r y acaba en 91v (ocupando, aproximadamente, unas 80 líneas), mientras que en $D$ se encuentra en las páginas 69-73. 
podido saber, y lo echo menos, y querría salir de este encanto». Vargas le dijo: «Tiempo hay, que agora voy averiguar cuál fue primero: la mentira o el sastre. Porque si la mentira fue primero, ¿quién lo pudo decir si no había sastres?; y si fueron primero los sastres, ¿¿cómo pudo haber sastres sin mentira? En averiguándolo esto volveré». Y en esto se desapareció $\mathrm{H}_{1} \mathrm{D}$ [ligeras diferencias entre si]

Entre los escritores conceptistas del Barroco, en general críticos con estas fórmulas del lenguaje, Quevedo es el «que mantiene una oposición mayor a todas estas muletillas. Los ataques se rastrean a través de su obra entera y durante toda su vida» (Arellano 8). No hay razones para pensar que el pasaje se deba a una persona diferente al autor; además, la presencia de temas y recursos retóricos propios de su estilo apuntan a la mano de Quevedo ${ }^{14}$. Asimismo, como también destaca Gónzalez López (1999: 162), este pasaje está presente en la versión de los Sueños contenida en Juguetes de la niñez y travesuras del ingenio (Madrid, 1631), edición autorizada por el propio Quevedo, lo que constituye un ulterior indicio de que puede tratarse de variante de autor, dado que, de ser apócrifa la interpolación, quizás la hubiera suprimido.

El hecho de que esta parte de texto falte en $A_{1}$ y en $B_{27}$ plantea dos posibilidades: en primer lugar que estuviese presente en el original y que se omitiese, después, en $A_{1}$ y en $B_{27}$-dos testimonios que, aparentemente, no guardan ninguna relación entre sí- o en alguno de sus antecedentes; en segundo lugar que faltase en la primera redacción, lo que significaría que el autor la habría ańadido posteriormente, aunque es imposible saber en qué momento de la transmisión. De todas maneras, no hay pruebas de que la ausencia del pasaje en $A_{1}$ y $B_{27}$ se deba a un error de copia o a una pérdida de hojas, porque en sus textos no se observan interrupciones en la contigüidad lógica o en la construcción sintáctica:

[...] y aparteme de él huyendo por no le ver aserrar con las costillas un paredón a puros carcomos. Íbame poco a poco ya buscando quien me guiase, cuando, sin hablar palabra, ni chistar, como dicen los nińos, un muerto de buena disposición, bien vestido y de buena cara, cerró conmigo $A_{1} B_{27}$ por no] y por no $B_{27}$ ya buscando] y buscando $B_{27}$

Obviamente, el hecho de que solo dos de los testimonios manuscritos conserven la parte final del texto limita las argumentaciones a favor de una $\mathrm{u}$ otra hipótesis; lo cierto es que el usus scribendi y el contenido del pasaje parecen no dejar duda en torno a la autoría quevediana.

En resumen, el texto del Sueño de la muerte contenido en Desvelos se acerca a la versión de los testimonios manuscritos, mientras que la única coincidencia relevante entre $D$ y $B_{27}$ es el error que se encuentra en la dedicatoria. Este dato sugiere que la persona que preparó la edición debía de tener a su disposición también una copia de la princeps. Sin embargo, tratándose de un error que se

14. A este propósito, ver Bertuzzi. Ningún editor ha expresado dudas sobre el carácter autorial del pasaje. Tanto Crosby (Quevedo 1993: 106) como Maldonado (Quevedo 1972: 236-241) lo insertan en el texto de sus respectivas ediciones del Sueños de la muerte basadas en $B_{27}$ (Crosby se sirve del texto de la princeps solo para la parte final del «sueńo», puesto que los manuscritos de los que disponía están incompletos). 
encuentra en la dedicatoria, este no constituye una prueba determinante para afirmar que el impreso barcelonés hubiera servido como texto base en el caso de Muerte.

Las VARIANTES De $D$ CON RESPECTO a $B_{27}$ Y A LOS TESTIMONIOS MANUSCRITOS

Como ya hubo ocasión de comentar, el texto del Sueño de la muerte editado en Desvelos contiene un alto número de cambios con respecto a la versión que ofrecen los manuscritos y la princeps. Los errores de copia se reducen a pocos casos de erratas, probablemente cometidas en el proceso de impresión, mientras que una pequeña cantidad de las lecturas privativas de $D$ son variantes de índole dudosa, porque podrían haber sido introducidas tanto consciente como inconscientemente. Entre ellas se registran sobre todo omisiones, como las que se reproducen en los ejemplos seleccionados:

Preguntele si era sastre, y él tan pronto me respondió: «No soy sino solicitador D

«Dígote sastre» dije yo. Y él tan presto dijo: «;Oir, que no pica!, pues no soy sino solicitador $\mathrm{B}_{27}$

«Dígole sastre» dije yo. Y tan presto dijo: «iOx, que no pica!, pues no soy sino solicitador $\mathrm{A}_{1} \mathrm{H}_{1} \mathrm{JPQ}$ [ligeras diferencias entre si]

un hombre viejo, ponderado de testuz, y de los que traen canas por vanidad D

un hombre viejo, muy ponderado de testuz, de los que traen canas por vanidad, una gran haz de barbas, ojos a la sombra, muy metidos, frenteaza llena de surcos, ceño descontento, vestido que juntando lo extraordinario con el desaliño, hacía misteriosa la pobreza $\mathrm{B}_{27} \mathrm{~A}_{1} \mathrm{H}_{1} J P Q$ [ligeras diferencias entre si]

En ambos casos las omisiones pudieron producirse tanto por un error de transcripción como por una supresión voluntaria por parte de alguien que, por ejemplo, no hubiese entendido la exclamación «¡Ox, que no pica!» o que hubiese querido recortar el texto. Otro ejemplo de variante de índole dudosa es el siguiente:

$\mathrm{Y}$ es de advertir que en todos los oficios, artes y estados se ha introducido el dar: en hidalgos, en villanos, en frailes, como se ve en la Cartuja. Yo he visto sastres y albañiles con don y labradores y galeotes en galera [...] Solo los médicos ninguno ha habido con don y todos tienen don de matar D

$\mathrm{Y}$ es de advertir que en todos los oficios, artes y estados se ha introducido el don: en hidalgos, en villanos, y en frailes, como se ve en la Cartuja. Yo he visto sastres y albañiles con don y ladrones y galeotes en galeras [...] Solo de los médicos ninguno ha habido con don y todos tienen don de matar $\mathrm{B}_{27} \mathrm{~A}_{1} \mathrm{H}_{1} J \mathrm{JQ}$ [ligeras diferencias entre sí]

La sustitución de don con dar puede deberse a un error por confusión gráfica, pero también a un cambio insertado sin entender el significado del pasaje, que, al mismo tiempo que satiriza el uso excesivo del título don, refuerza la crítica contra los médicos. La variante dar constituye, por lo tanto, una lectura peor con respecto a don. 
Los casos que se han considerado representan solo una pequeña parte de las lecturas privativas de $D$, porque la gran mayoría de sus variantes se introdujeron con la clara intención de modificar el texto, lo que demuestra que fue sometido a una verdadera revisión antes de ser publicado. La revisión que, en principio, pudo ser llevada a cabo por una o más personas, toca diferentes aspectos del texto, por lo que he dividido las variantes en grupos según el componente textual al que afectan. Forman parte del primer grupo aquellos cambios insertados con el objetivo de mejorar la conexión textual en diferentes niveles. Para empezar, algunas modificaciones afectaron a las relaciones sintácticas:

En esto estaba cuando se oyó una voz que dijo tres veces: «iMuertos, muertos, muertos!». Al punto se rebulló el suelo y todas las paredes D

En esto estaba cuando se oyó una voz que dijo tres veces: «iMuertos, muertos, muertos!». Con eso se rebulló el suelo y todas las paredes $\mathrm{B}_{27} \mathrm{~A}_{1} \mathrm{H}_{1} \mathrm{JPQ}$ [ligeras diferencias entre sí]

dijo la Muerte: «Hablen por su antigüedad». Dado este decreto salió uno con grandísima cólera y priesa, y se vino para mí, de suerte que entendí me quería maltratar D

«Hablen por su orden», dijo la Muerte. Luego salió uno con grandísima cólera y priesa, y se vino para mí, que entendí que me quería maltratar $\mathrm{B}_{27} \mathrm{~A}_{1} \mathrm{H}_{1}$ JPQ [ligeras diferencias entre si]

soy el rey Perico, y no me dejan descansar de día y de noche, porque no hay cosa sucia, desaliñada, pobre, antigua, ni mala, que no digan luego «Fue en tiempo del rey Perico» D

soy el rey Perico, y no me dejan descansar de día ni de noche. No hay cosa sucia ni desaliñada, ni pobre, ni antigua, ni mala, que no digan que fue en tiempo del rey Perico $\mathrm{B}_{27} \mathrm{~A}_{1} \mathrm{H}_{1} \mathrm{JPQ}$ [ligeras diferencias entre si]

Estos cambios se limitan a la inserción o sustitución de conjunciones y demás elementos de conexión sintáctica por otros que tienen el objetivo de explicitar la relación entre cláusulas, pero también de aportar mayor variedad en los conectores textuales. En lo que se refiere a la variedad, en el primer ejemplo es probable que "con eso/esto» se sustituyera para evitar la repetición del pronombre demostrativo, ya utilizado al principio de la frase («En esto»). Esta impresión es apoyada otras veces por la sustitución de la conjunción $y$ por otros conectores:

[médicos:]

sino que como han oído decir "Quien no te conoce te compre», disfrazan las legumbres y las demás cosas de que se valen, porque no sean conocidas y las compren los enfermos $\mathrm{D}$

$y$ como han oído decir que «Quien no te conoce te compre», disfrazan las legumbres, porque no sean conocidas y las compren los enfermos $\mathrm{B}_{27} \mathrm{~A}_{1} \mathrm{H}_{1}$ JPQ [ligeras diferencias entre si]

No me espantó; pero suspendiome, aunque no sin risa, porque bien mirada era figura donosa $\mathrm{D}$ 
No me espantó; suspendiome, $y$ no sin risa, porque bien mirado era figura donosa $\mathrm{B}_{27} \mathrm{~A}_{1} \mathrm{H}_{1} \mathrm{JPQ}$

Asimismo, en la versión de Desvelos se registran una serie de añadidos que enriquecen y refuerzan la conexión entre párrafos y entre periodos sintácticos:

las más veces de asco de las porquerías y hediondeces con que persiguen a los enfermos se huyen las enfermedades. $O$, si no, pregunto, ¿qué dolor habrá de tan mal gusto, que no se huya D

las más veces de asco de sus porquerías y hediondeces con que persiguen a los enfermos se huyen las enfermedades. ¿Qué dolor habrá de tan mal gusto que no se huya $\mathrm{B}_{27} \mathrm{~A}_{1} \mathrm{H}_{1} \mathrm{JPQ}$ [ligeras diferencias entre si]

\section{[Muerte:}

conmigo nadie va vestido, ni soy embarazosa. Yo traigo los trastes de todos, porque vayan más ligeros. Apresteme pues viendo esto, y fui con ella donde me guiaba D

conmigo nadie va vestido, ni soy embarazosa. Yo traigo los trastos de todos, porque vayan más ligeros. Fui con ella donde me guiaba $\mathrm{B}_{27}$

conmigo nadie va vestido, ni soy embarazosa. Yo traigo los trastes de todos porque vayan más ligeros. Apresteme y fui con ella adonde me guiaba $\mathrm{A}_{1} \mathrm{H}_{1} \mathrm{JPQ}$

\section{[don Diego de Noche:]}

los gusanos se mueren de hambre conmigo y aun yo me como a los gusanos de hambre. Mas no es este mi mayor mal, sino que los muertos andan huyendo de mí D

los gusanos se mueren de hambre conmigo y yo me como a los gusanos de hambre, y los muertos andan siempre huyendo de mí $\mathrm{B}_{27} \mathrm{~A}_{1} \mathrm{H}_{1}$ siempre] om. $\mathrm{A}_{1} \mathrm{H}_{1}$

Las adiciones introducen al principio de una frase elementos de referencia al texto que la precede, reforzando así la conexión con lo dicho anteriormente. Además, en algunas ocasiones los añadidos contienen elementos que expresan los sentimientos y actitudes del narrador o de otro personaje de Muerte:

Con estas consideraciones divertido, me aparté sin sentir de ambos, y bajamos a un grandísimo llano D

Con esto bajamos a un grandísimo llano $\mathrm{B}_{27} \mathrm{~A}_{1} \mathrm{H}_{1} \mathrm{JPQ}$

Propósitos similares a los que se observan en las variantes que modifican la conexión textual (para crear mayor variedad lingüística y desarrollar la transición de un enunciado a otro) emergen también de los cambios que afectan a los diálogos. Estas partes del texto fueron sometidas a una revisión que concierne sobre todo a los elementos que acompañan el discurso directo, empezando por sustituciones recurrentes en los verba dicendi, que parecen 
haberse insertado para evitar el abuso del verbo decir, sustituido sobre todo con replicar y responder, según el contexto ${ }^{15}$.

Pero las modificaciones más relevantes en los diálogos son las adiciones de elementos circunstanciales que enmarcan el discurso directo, tanto en lo que concierne al estado del hablante como a su entorno. A veces estos cambios están acompañados también por variantes que, dentro y fuera de las afirmaciones de los personajes, desarrollan la conexión sintáctica, lo que lleva a pensar que la persona que se ocupó de los diálogos debió de ser la misma que modificó la conexión textual en los casos analizados anteriormente. Se han elegido los ejemplos más significativos:

Miré al primero con atención, y me pareció notable cosa. La Muerte, reparando en mi cuidado, me dijo: «¿Qué miras?», y yo la respondí: «Miro al infierno y me parece que le he visto mil veces» $\mathrm{D}$

Estuve mirando al infierno con atención y me pareció notable cosa. Díjome la Muerte: "QQué miras?». «Miro -respondí- al infierno, y me parece que le he visto tres veces» $\mathrm{B}_{27} \mathrm{~A}_{1} \mathrm{H}_{1} \mathrm{JPQ}$ [ligeras diferencias entre si]

Estos son gente que están en el otro mundo, y aún no se persuaden a que son difuntos. Maravillome esta visión al fin sobre todas y, viendo estas y otras mil diferencias, herido del dolor y conocimiento, dije $\mathrm{D}$

Estos son gente que están en el otro mundo, y aún no se persuaden a que son difuntos. Maravillome esta visión, y dije, herido del dolor y conocimiento $\mathrm{B}_{27} \mathrm{~A}_{1} \mathrm{H}_{1}$ JPQ [ligeras diferencias entre si]

"Con todo esto no he dicho lo que siento, y así quiero, como no te enfades, decirte que tan malos son». «Holgareme mucho» dijo el marqués. «Pues son tan malos -dije yo- que, si no hubiera letrados, no hubiera porfías D

«Queréis ver qué tan malos son los letrados? Que si no hubiera letrados, no hubiera porfías $\mathrm{B}_{27} \mathrm{~A}_{1} \mathrm{H}_{1} \mathrm{JPQ}$ los] om. J

El marqués, lastimado de semejante ruina y miseria, dijo entonces: «iTente, no pases adelante!, que no he de salir de aquí hasta que los pleitos se determinen a garrotazos $\mathrm{D}$

No he de salir de aquí -dijo el nigromántico- hasta que los pleitos se determinen a garrotazos $\mathrm{B}_{27} \mathrm{~A}_{1} \mathrm{H}_{1} \mathrm{JPQ}$ [ligeras diferencias entre si]

Mientras yo le estaba oyendo con atención, muy enojado y melancólico, se llegó a mí un hombre viejo D

15. Como, por ejemplo, en el pasaje que se reproduce a continuación:

"¿Quién es vuestra merced -respondí yo entonces-, que con tanto imperio habla y donde todos son iguales presume diferencias?». "Yo soy -replicó el difunto- el Rey que Rabió D

"¿Quién es vuestra merced - dije yo-, que con tanto imperio habla y donde todos son iguales presume diferencia?». «Yo soy -dijo- el Rey que Rabió $\mathrm{B}_{27} \mathrm{~A}_{1} \mathrm{H}_{1} J \mathrm{PQ}$ [ ligeras diferencias entre si] 
Muy enojado a mí se llegó un hombre viejo $\mathrm{B}_{27}$

Muy enojado y melancólico se llegó a mí un hombre viejo $\mathrm{A}_{1} \mathrm{H}_{1} \mathrm{JPQ}$

Con respecto a la versión de la princeps y de los manuscritos, en la que la interacción entre los personajes no suele tener otra interrupción sino el verbum dicendi y la identificación del hablante, en Desvelos los diálogos asumen un ritmo más lento. En general, las adiciones de $D$ añaden información sobre el estado del hablante; sin embargo, no se trata de aportaciones sustanciales para el texto. De hecho, en muchos casos, la adición de $D$ no hace más que reiterar conceptos que ya se expresan en el texto tal y como aparece en los demás testimonios. Tómese, por ejemplo, la primera de las variantes citadas arriba: el narrador dice que está mirando el infierno con atención y en $D$ se añade que la muerte repara en su cuidado. De la misma manera, en el penúltimo caso citado, se especifica que el marqués se siente lastimado «de semejante ruina y miseria», un estado que sus palabras ya transmiten en todas sus afirmaciones. Por lo tanto, me parece que los cambios introducidos en los diálogos -que resultan en gran medida modificados- responden, entre otras cosas, a la necesidad de ofrecer un texto de comprensión más inmediata para el lector.

Otro grupo de variantes de $D$ está constituido por cambios en la estructura del periodo, obtenidos a través de desplazamientos. Veamos un primer ejemplo:

sin que haya habido rey de tan mala asquerosa carroña, caduca, carcomida y apolillada memoria en el mundo, pues no se acuerdan de él sino vejeces, harapos, antigüedades $\mathrm{y}$ visiones. Yo en efeto estoy aquí con esta nota, porque todos han dado en decir que rabié, y juro a $\mathrm{N}$. que mienten si bien ya no tiene remedio. Solo me consuela considerar no hay rey ni le ha habido ni le habrá a quien no levanten que rabia $\mathrm{D}$

No ha habido tan desdichado rey en el mundo, pues no se acuerdan de él sino vejeces, y harapos, antigüedades y visiones, y ni ha habido rey de tan mala memoria ni tan asquerosa, ni tan carroña, ni tan caduca, carcomida y apolillada. Han dado en decir que rabié y juro a Dios que mienten, sino que han dado todos en decir que rabié y no tiene ya remedio, y no soy yo el primero rey que rabió ni el solo, que no hay rey ni le ha habido ni le habrá a quien no levanten que rabié $\mathrm{B}_{27} \mathrm{~A}_{1} \mathrm{H}_{1}$ JPQ [ligeras diferencias entre si]

En este pasaje, junto al cambio de estructura, se encuentran otras variantes que muestran características ya observadas y que parecen propias del usus scribendi de la persona a la que se deben la mayoría de las modificaciones presente en $D$. En lo que concierne al cambio en la estructura sintáctica, se efectuaron un desplazamiento y una refundición. En la versión de $B_{27}$ y de los testimonios manuscritos el orden del periodo sintáctico está constituido por la cláusula principal, la subordinada causal (introducida por pues) y la coordinada con la principal; mientras que en Desvelos se funden las dos cláusulas coordinadas en una sola, a la cual sigue la causal. Este desplazamiento incluye un cambio de posición del sustantivo memoria, que pasa del principio al final de la cláusula, donde la relación lógica con la causal resulta más inmediata. Este tipo de cambio se hizo con la finalidad de mejorar tanto la linealidad del texto como su conexión interna (en este pasaje también hay modificaciones que desarrollan los elementos que encadenan las frases). Al mismo tiempo, con los cambios se quieren evitar las repeticiones. 
Esta manera de operar, a través de desplazamientos acompañados de sustituciones y de la introducción de nuevos elementos, se empleó en otros lugares del texto. Los ejemplos que se reproducen abajo se han seleccionado por ser especialmente significativos en relación con el problema de la autoría de las variantes:

lo que más he estimado es haber visto el Juicio, porque ahora que veo como es, echo de ver que el que hay en el mundo no es juicio ni hay hombre que le tenga $\mathrm{D}$

lo que más he estimado es haber visto el Juicio, porque hasta agora he vivido engañado, y agora que veo al juicio como es, echo de ver que el que hay en el mundo no es juicio ni hay hombre de juicio y que hay muy poco juicio en el mundo $\mathrm{B}_{27} \mathrm{~A}_{1} \mathrm{H}_{1}$ JPQ [ligeras diferencias entre si]

Todos tienen honra, y todos los estados son honrados, y todo se hace todo caso de honra, y la honra se está cayendo de su estado y aun parece está ya siete estados debajo de tierra D

Todos tienen honra, y todos son honrados, y todos lo hacen todo caso de honra. Hay honra en todos estos estados, y la honra se está cayendo de su estado y parece que está ya siete estados debajo de tierra $\mathrm{B}_{27} \mathrm{~A}_{1} \mathrm{H}_{1} \mathrm{JPQ}$ [ligeras diferencias entre si]

En ambos casos el texto de Desvelos presenta una versión reducida de la frase con respecto a como aparece en los demás testimonios. Quien introdujo los cambios quiso eliminar las que parecen repeticiones y ordenar la sintaxis con el objetivo de obtener un texto más lineal. Sin embargo, tanto en el primero como en el segundo caso, la repetición de la palabra juicio antes y honra después crea un juego de palabras, porque «Quevedo reitera dos o más veces una palabra en la frase con significado distinto en cada caso. La iteración enfática del vocablo en cuestión acentúa el contraste de sentidos» (Schwartz 1986: 27). En lo que se refiere a la reiteración de juicio «Quevedo se burla del sistema de justicia en su sociedad, de la falta de sentido común en el hombre y de la falta de justicia o equidad ${ }^{16}$. A continuación, el autor juega con tres acepciones de la palabra estado, utilizada, primero, con el sentido de 'estamento del cuerpo social'; después, en la frase 'caer de su estado', adquiere el significado de 'perder su valimiento' y, en la tercera recurrencia, se usa con el valor de 'medida' (Autoridades). En ambos casos Quevedo crea una antanaclasis, figura retórica otras veces utilizada por el autor ${ }^{17}$. Por lo tanto, estas variantes de $D$ constituyen un empeoramiento del texto y no pueden atribuirse a Quevedo.

En la versión de Muerte que ofrece Desvelos se encuentran ulteriores desplazamientos con respecto al orden en el que se disponen algunos elementos del texto en los demás testimonios. En general, los cambios responden a las mismas necesidades que están detrás de los dos casos que se acaban de considerar: crear un periodo con mayor cohesión y linealidad, además de evitar repeticiones de la misma palabra. De todos ellos, el caso más relevante, por

16. Así lo explica Crosby en la nota referida a este pasaje en su edición de los Sueños (Quevedo 1993 : 1418).

17. Por ejemplo, en el Buscón y en el Caballero de la Tenaza, cf. Schwartz. 
suponer una alteración notable de estructura en el texto, se sitúa en la parte final del «sueño» y concierne al episodio en el que el narrador encuentra al personaje don Diego de Noche. En el texto de todos los testimonios excepto $D$ el narrador lo hace en dos ocasiones diferentes, mientras que en Desvelos solamente una vez, al fundirse los dos episodios en uno:

\begin{tabular}{|c|c|}
\hline $\operatorname{Mss}\left(A_{l}\right.$ y $\left.H_{1}\right)$ y $B_{27}$ & Desvelos \\
\hline $\begin{array}{l}\text { [Dueña Quintañona]: «estoy muy } \\
\text { vieja para andar en refranes y quería } \\
\text { más andar en zancos, porque no deja de } \\
\text { cansar a una persona andar de boca en } \\
\text { boca» }\end{array}$ & $\begin{array}{l}\text { [Dueña Quintańona]: "que hagáis } \\
\text { metan otra dueña en el refrán y me dejen } \\
\text { descansar, que estoy muy vieja para andar } \\
\text { en refranes y querría más andar en zancos» }\end{array}$ \\
\hline $\begin{array}{l}\text { Muy angosto, muy a teja vana las } \\
\text { carnes, devanado en un cendal }[\ldots] \\
\text { Preguntele quién era: «Yo soy el malcosido } \\
\text { y peor sustentado don Diego de Noche» }\end{array}$ & $\begin{array}{l}\text { Desapareciose con esto aquella patarata } \\
\text { infernal, porque a los muertos dio gana } \\
\text { de comer } \\
\text { [Otros personajes: Cochitehervite, } \\
\text { Trochimochi, ..., Santo de Pajares] }\end{array}$ \\
\hline $\begin{array}{l}\text { Desapareció aquel caballero y visión, } \\
\text { y dio gana de comer a los muertos: } \\
\text { cuando llegó a mi [...] } \\
\text { [Otros personajes: Cochitehervite, } \\
\text { Trochimochi, ..., Santo de Pajares] }\end{array}$ & \multirow{2}{*}{$\begin{array}{l}\text { Yo, creyendo era ya todo acabado, volví } \\
\text { las espaldas y topeme cosido conmigo a un } \\
\text { muerto muy angosto, y muy a teja vana, las } \\
\text { carnes de venado [...] Preguntele quién era } \\
\text { y respondió: "Yo soy el malcosido y peor } \\
\text { sustentado don Diego de Noche» }\end{array}$} \\
\hline $\begin{array}{l}\text { Volví las espaldas y topé cosido } \\
\text { conmigo don Diego de Noche, } \\
\text { rascándose en una esquina; conocile y } \\
\text { díjele: «Es posible que aún hay que comer } \\
\text { en vuestra merced, señor don Diego?» } \\
\text { [...] Diome grande risa y aparteme dél } \\
\text { huyendo, y por no le ver aserrar con las } \\
\text { costillas un paredón a puros carcomos }\end{array}$ & \\
\hline
\end{tabular}

En el texto de $B_{27}$ y de los manuscritos don Diego de Noche aparece después del personaje de la dueña Quintañona; el fragmento acaba con estas palabras: «Desapareció aquel caballero y visión, y dio gana de comer a los muertos». Aparecen entonces otros personajes, empezando por Cochitehervite, hasta que, finalmente, vuelve don Diego de Noche. En Desvelos, en cambio, después del episodio de la dueña se introduce a Cochitehervite y la lista de personajes que éste encabeza; cuando el último de ellos se ha ido, se introduce a don Diego de Noche.

El cambio de orden y la fusión de las dos partes en una requirió algunos ajustes en el texto; uno de ellos resulta especialmente significativo: se trata de la frase con la que se va la dueña en $D$, que es una reelaboración de las palabras 
con las que desaparece Diego de Noche en $B_{27} A_{l}$ y $H_{l}$. La imagen de «dar ganas de comer a los muertos» no tiene sentido en la versión de $D$, porque se asocia al personaje de la dueńa, cuando es en realidad Diego de Noche quien se satiriza como hambriento; por lo tanto, como ya hizo notar Crosby: «la referida transposición despojó a estas palabras de su razón de ser» (Quevedo 1993: $104-105)^{18}$.

Las lecturas privativas del texto de Desvelos que se han considerado hasta ahora parecen todas imputables a una sola persona por mostrar elementos comunes atribuibles a un mismo usus scribendi. La persona que se ocupó de estos cambios fue la que insertó las variantes más importantes, porque se trata de alteraciones que modifican el texto de manera profunda. Como se fue destacando a lo largo del análisis, si algunas de estas variantes conllevan cambios aceptables, otras empeoran el texto de manera evidente, lo que demuestra que la mayoría de las lecturas privativas del texto de Desvelos fueron introducidas por una persona distinta del autor.

Aparte de las variantes analizadas quedan todavía algunas lecturas privativas que no muestran de manera evidente esas características que permitirían atribuirlas a la misma persona, aunque eso no excluye que también procedan de su mano. Para empezar, hay una serie de variantes que crean un texto más explícito, algo que se consigue a través de dos tipos de cambios. En primer lugar, se encuentran lecturas que explicitan el estilo limitándose a pequeñas modificaciones, sin alterar el significado de la frase o aportar nuevos elementos al texto:

Fueron entrando al principio unos médicos a caballo en unas mulas, tales que parecían con sus gualdrapas negras tumbas con orejas D

Fueron entrando unos médicos a caballo en unas mulas, que con gualdrapas negras parecian tumbas con orejas $\mathrm{B}_{27} \mathrm{~A}_{1} \mathrm{H}_{1} \mathrm{JPQ}$

Y es cosa notable que todos se parecían unos a otros sin que se diferenciasen en cosa alguna $\mathrm{D}$

$\mathrm{Y}$ es cosa notable que eran todos parecidos unos a otros que no se diferenciaban $\mathrm{B}_{27} \mathrm{~A}_{1} \mathrm{H}_{1} \mathrm{JPQ}$ es] era $\mathrm{P}$ eran todos parecidos] eran tan parecidos $\mathrm{H}_{1}$ parecian $\mathrm{P}$ que] y $\mathrm{A}_{1}$

cualquier ruido que el viento hace $\mathrm{D}$

cualquier ruido del viento $\mathrm{B}_{27} \mathrm{~A}_{1} \mathrm{H}_{1} \mathrm{JPQ}$

En segundo lugar, se encuentran variantes que explicitan el significado de un pasaje a través de la adición de nuevos elementos:

[genoveses:]

18. Arellano hace una observación parecida a propósito de esta variante de $D$ en un comentario al aparato crítico de su edición basada en $B_{27}$ (Quevedo 2007: 599). González López (1999: 161), en cambio, opina: «La perfecta coherencia con que se ha reelaborado $D$ (ensambla perfectamente en una nueva redacción frases y motivos dispersos en $B$ ) se diría excesivo atribuirla a la labor de copistas o de alguien ajeno a su autor» (con la sigla $B$ se refiere a la princeps). 
hecho polvos en salvadera quiero estar antes que verlos hechos dueños de todo». «Señor marqués -repliqué yo-, aunque eso es así, han dado de suerte en adolecer de caballeros, que no hay que temerlos mucho. En teniendo caudal, se untan luego en señores y enferman de príncipes $\mathrm{D}$

hecho polvos en salvadera quiero estar antes que verlos hechos dueños de todo». «Señor nigromántico -repliqué yo-, aunque esto es ansí, han dado en adolecer de caballeros en teniendo caudal, úntanse de señores y enferman de príncipes $\mathrm{B}_{27} \mathrm{~A}_{1} \mathrm{H}_{1}$ JPQ [ligeras diferencias entre si]

\section{[letrados:]}

Ahora ha entrado la cáfila de Menoquios, Surdos y Fabros, Farinacios y Cujacios, consejos, decisiones, responsiones, lectiones y meditaciones, con que se ha confundido todo. Y aun si parara en esto fuera menos mal, pero cada día salen autores, cada uno con una infinidad de volúmenes $\mathrm{D}$

Ahora ha entrado una cáfila de Menoquios, Surdos y Fabros, Farinacios y Cujacios, consejos y decisiones y responsiones y lectiones y meditaciones, y cada día salen autores, y cada uno con tres volúmenes $\mathrm{B}_{27} \mathrm{~A}_{1} \mathrm{H}_{1} \mathrm{JPQ}$ [diferencias entre si]

¿Pues qué, si vais a la platería? No volveréis enteros. Allí cuesta la estimación, la honra, y hay quien hace encreyente a un malaventurado, que se cińa todo su patrimonio al dedo, poniéndole un sortijón de vidrios con nombre de diamantes. Y no sintiendo los artejos el peso, está aullando su casa D

¿Pues qué, si vais a la platería? No volveréis enteros. Allí cuesta la estimación, la honra, y hay quien hace encreyente a un malaventurado que se cińa su patrimonio al dedo, y no sintiendo los artejos el peso, está aullando en su casa $\mathrm{B}_{27} \mathrm{~A}_{1} \mathrm{H}_{1} \mathrm{P}$ [ [igeras diferencias entre si]

Lo que se expresa en las variantes de $D$ queda implícito en la versión del texto brindada por la princeps y por los testimonios manuscritos. Sin embargo, en $D$ se explicita algo que puede fácilmente inferirse del contexto, por lo que no se trata de adiciones significativas. Hay otros lugares en los que en $D$ se clarifica el texto a través de breves añadidos insertados:

No hay gente más fiera que estos boticarios, son armeros de los dotores, ellos les dan las armas con que nos matan $\mathrm{D}$

No hay gente más fiera que estos boticarios, son armeros de los dotores, ellos les dan armas $\mathrm{B}_{27} \mathrm{~A}_{1} \mathrm{H}_{1}$ JPQ les] los J

¡Oh malditos pesquesidores contra la vida humana! D

¡Oh malditos pesquisidores contra la vida! $\mathrm{B}_{27} \mathrm{~A}_{1} \mathrm{H}_{1} \mathrm{JPQ}$

Considerando, pues, yo la diferencia grande del acompańamiento y no sabiendo imaginar quién pudiese venir tras tanta máquina de gente tan diversa en oficios y humores $\mathrm{D}$

En esto estaba yo considerando la diferencia tan grande del acompańamiento y no sabía imaginar quién pudiese venir $\mathrm{B}_{27} \mathrm{~A}_{1} \mathrm{H}_{1} \mathrm{JPQ}$ imaginar] om. $\mathrm{A}_{1}$ 
Todos los ejemplos citados en los que se reproducen cambios que tienen la finalidad de explicitar o aclarar el texto constituyen variantes equipolentes aceptables en el contexto en el que se insertan. No puede decirse con seguridad que estas variantes fueron introducidas por la misma persona que revisó el texto en los casos analizados precedentemente, pero es improbable que se trate de cambios que resultaron de intervenciones del autor, porque no son significativos para el texto y no muestran rasgos del usus scribendi de Quevedo, razón por la cual cualquiera habría podido introducirlos.

Finalmente, en el texto de $D$ se encuentran algunas variantes de carácter paliativo. En el diálogo que el narrador mantiene con el marqués de Villena se sustituyeron todas las referencias a la República de Génova, llamada simplemente «cierta república». El término genoveses también se sustituye con referencias de carácter genérico (aunque por el contexto sigue siendo fácil adivinar el sujeto de la conversación):

«¿Extranjeros andan a la zacapela con el dinero? -dijo el marqués- ¡Vuélvome jigote! Hijo mío, los hombres de negocios (estos de cambios y asientos) son lamparones del dinero, enfermedad que procede de tratar con gatos; y vese que son lamparones porque solo el dinero que va a Francia sana de esos lamparones, por no admitir el rey cristianísimo semejante gente en su comercio D

"¿Ginoveses andan a la zacapela con el dinero? - dijo él- ¡Vuélvome jigote! Hijo mío, los ginoveses son lamparones del dinero, enfermedad que procede de tratar con gatos; y vese que son lamparones porque solo el dinero que va a Francia no admite ginoveses en su comercio $B_{27}$

«¿Genoveses andan a la zacapela con el dinero? -díjome el marqués- ¡Vuélvome jigote! Hijo mío, los genoveses son lamparones del dinero, enfermedad que procede de tratar con gatos; y vese que son lamparones porque solo el dinero que va a Francia sana de esos lamparones, porque el rey de Francia no admite genoveses en su comercio $\mathrm{A}_{1} \mathrm{H}_{1} \mathrm{JPQ}$ [diferencias entre sí]

Lo curioso de estos cambios es que se centran exclusivamente en los genoveses y no, por ejemplo, en los venecianos, de los cuales se habla justo después. Ello se debe, quizás, a que en el siglo XVII la república genovesa mantenía fuertes lazos financieros con la monarquía hispánica, razón por la cual alguien consideraría las críticas como demasiado atrevidas. De todas maneras, no deja de llamar la atención el hecho de que se centrase la censura en este aspecto, sobre todo porque a lo largo del texto casi no se encuentran otras variantes paliativas claras.

En el pasaje sobre los genoveses, en $D$ se sustituyó también "rey de Francia», como aparece en los manuscritos, por «rey cristianísimo», «renombre que por antonomasia se daba solo a los reyes de Francia» (Autoridades). Por lo demás, se encuentran algunas omisiones que, muy probablemente, se deben asimismo a razones de censura, porque en $D$ se suprime un pasaje de cinco líneas en el que los españoles se retratan como borrachos y putos («Honrados eran los españoles cuando podían decir putos y borrachos a los extranjeros [...] Ahora me dicen que los culos se han introducido en barrigas»); y otra referencia a la sodomía 
en el pasaje sobre los venecianos («la sodomía en el fuego»). Otra vez, no hay elementos que permitan entender quién censuró estos pasajes.

En suma, de las variantes más significativas introducidas en el Sueño de la muerte de $D$ emergen características comunes en el usus scribendi y en las finalidades, que hacen pensar que por lo menos la mayoría de las modificaciones presentes proceden de la mano de una misma persona. En particular, su estilo busca evitar la repetición, mejorar la conexión textual y aclarar el texto. En muchos casos, las modificaciones introducidas son aceptables: quien revisó el texto lo hizo con cierto cuidado, preocupándose de respetar el sentido y el contenido, lo que atestigua sus capacidades literarias. No obstante, en diferentes ocasiones sus intervenciones tienen el efecto de empeorar el texto (por ejemplo, sus intentos de eliminar repeticiones de palabras lo llevó a suprimir un recurso retórico propio del usus scribendi de Quevedo, la antanaclasis). Por estas razones es improbable que el texto de Muerte contenido en Desvelos constituya una nueva versión atribuible a Quevedo. A la luz de lo que afirma Lorenzo Van der Hammen en la carta nuncupatoria que se incluye en la edición zaragozana y considerando su actividad literaria, lo más lógico es pensar que fue él quien llevó a cabo esta revisión ${ }^{19}$.

\section{Bibliografía}

Arellano Ignacio, «Notas sobre el refrán y la fórmula coloquial en la poesía burlesca de Quevedo", Rilce. Revista de filología hispánica, 1985, vol. 1, n 1, pp.7-31.

Azaustre Galiana Antonio, «La prosa burlesca de Quevedo y el Buscón», en Raphaël Carrasco (coord.), Le roman picaresque. La vida de Lazarillo de Tormes. Francisco de Quevedo, La vida del Buscón llamado don Pablos, Paris, Ellipses Édition, 2006, pp. 163-182.

Bertuzzi Irene, «Un ejemplo de manipulación textual realizada por un editor: la edición barcelonesa de los Sueños de 1628», La Perinola, 2014, n 18, pp. 235-253.

Blecua Alberto, Manual de crítica textual, Madrid, Castalia, 1990.

Díaz Martínez Eva María, "Prólogo», en Francisco de Quevedo, Política de Dios, gobierno de Cristo, en Alfonso Rey (dir.), Obras completas en prosa, vol. V, Madrid, Castalia, 2012, pp. 161-186.

González López Beatriz, La transmisión textual del «Sueño de la muerte» en las ediciones de 1627 y 1628, Memoria de Licenciatura, Santiago de Compostela, 1994.

— «Hacia una edición de los Sueños: Desvelos soñolientos», La Perinola, 1999, n³, pp. 157-170.

Jauralde Pou Pablo, Francisco de Quevedo (1580-1645), Madrid, Castalia, 1998.

Lázaro Carreter Fernando, «Estudio preliminar», en Francisco de Quevedo, La vida del Buscón llamado don Pablos, Salamanca, CSIC, 1965, pp.XI-LXXVIII.

19. Sin embargo, eso no implica que también el Sueño del Juicio y el Sueño del infierno hayan sufrido un proceso de preparación igual al de Muerte: de hecho, cada uno de los textos presenta frente a los demás testimonios un escenario peculiar. En el Sueño del infierno, por ejemplo, se encuentran algunos amplios añadidos que parecen atribuibles a Quevedo. A este propósito, ver Bertuzzi (243-246). 
Nolting-Hauff Ilse, Visión, sátira y agudeza en los «Sueños» de Quevedo, Madrid, Gredos, 1974.

Moll Jaime, De la imprenta al lector. Estudios sobre el libro español de lo siglos XVI al XVIII, Madrid, Arco Libros, 1994.

Quevedo Francisco de, «A don Lorenço van der Hammen y Leon, Vicario de Iubiles», en Lorenzo van der Hammen, Don Filipe el prudente, segundo deste nombre, rey de las Españas y Nuevo Mundo, en Madrid por la viuda de Alonso Martín, 1633.

- Sueños y discursos, edición de Felipe C. R. Maldonado, Madrid, Castalia, 1972.

- Obra poética, edición de José Manuel Blecua, Madrid, Castalia, 1969-1981, 4 volúmenes.

- Sueños y discursos, edición de James O. Crosby, Madrid, Castalia, 1993, 2 volúmenes.

- Discurso de todos los diablos, o infierno emendado, edición de Alfonso Rey, en Alfonso Rey (dir.), Obras completas en prosa, vol. I, t. 2, Madrid, Castalia, 2003a, pp. 469560.

- La Fortuna con seso y la Hora de todos. Fantasía moral, edición de Lía Schwartz, en Alfonso Rey (dir.), Obras completas en prosa, vol. I, t. 2, Madrid, Castalia, 2003b, pp. 561-810.

- El chitón de las tarabillas, edición de Manuel Ángel Candelas Colodrón, en Alfonso Rey (dir.), Obras completas en prosa, vol. III, Madrid, Castalia, 2005, pp. 185-247.

— Los sueños, edición de Ignacio Arellano, Madrid, Cátedra, 2007 (1ª edición 1991).

Rey Alfonso, "Quevedo, Duport y la edición del Buscón», Journal of Hispanic Research, 1994-5, n³, pp. 167-179.

Rodríguez Cáceres Milagros, «El manuscrito Aldecoa: un testimonio desconocido y completo del Sueño de la muerte», La Perinola, 2007, n 11, pp. 227-257.

- "Hospedería reales, otro manuscrito desconocido de los Sueños de Quevedo», La Perinola, 2008, n 12, pp. 373-390.

Schwartz Lía, Metáfora y sátira en la obra de Quevedo, Madrid, Taurus, 1983.

- Quevedo: discurso y representación, Pamplona, EUNSA, 1986.

Véliz Zahira y García Valverde María Luisa, «Don Lorenzo van der Hammen y León: vida en la Corte y en el exilio en el Siglo de Oro espańol», Reales sitios. Revista del partimonio nacional, 2006, vol.XLIII, n ${ }^{\circ}$ 67, pp. 3-27.

Van der Hammen Lorenzo, "A don Francisco Jiménez de Urrea, Capellán de su Majestad», en Francisco de Quevedo, Los Sueños, edición de Ignacio Arellano, Madrid, Cátedra, 2007. 